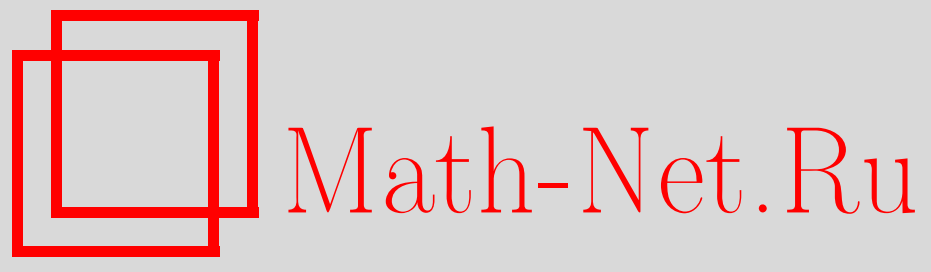

П. А. Бородин, $\mathrm{K}$ задаче существования элемента с заданными уклонениями от расширяющейся системы подпространств, Матем. заметки, 2006, том 80, выпуск $5,657-667$

DOI: https://doi.org/10.4213/mzm3075

Использование Общероссийского математического портала Math-Net.Ru подразумевает, что вы прочитали и согласны с пользовательским соглашением http://www . mathnet.ru/rus/agreement

Параметры загрузки:

IP : 54.196 .121 .252

26 апреля 2023 г., 08:32:19

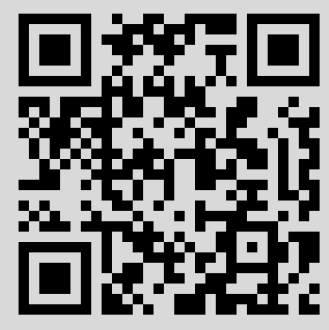




\section{К ЗАДАЧЕ СУЩЕСТВОВАНИЯ ЭЛЕМЕНТА \\ С ЗАДАННЫМИ УКЛОНЕНИЯМИ \\ ОТ РАСШИРЯЮЩЕЙСЯ СИСТЕМЫ ПОДПРОСТРАНСТВ}

\section{П. А. Бородин}

Задача о существовании элемента банахова пространства, имеющего заданные уклонения от расширяющейся системы строго вложенных (необязательно конечномерных) подпространств этого пространства, решается при дополнительных ограничениях на уклонения или на подпространства.

Библиография: 12 названий.

Указанная в заголовке задача формулируется так: пусть задана счетная система $Y_{1} \subset Y_{2} \subset Y_{3} \subset \cdots$ строго вложенных линейных подпространств некоторого бесконечномерного банахова пространства $(X,\|\cdot\|)$, полная в $X$ :

$$
\overline{\bigcup_{n=1}^{\infty} Y_{n}}=X \text {, }
$$

а также последовательность неотрицательных чисел $d_{1} \geqslant d_{2} \geqslant d_{3} \geqslant \cdots, d_{n} \rightarrow 0$. Существует ли элемент $x \in X$, уклонения $\rho\left(x, Y_{n}\right):=\inf \left\{\|x-y\|: y \in Y_{n}\right\}$ которого от подпространств $Y_{n}$ равны этим числам:

$$
\rho\left(x, Y_{n}\right)=d_{n}, \quad n=1,2, \ldots ?
$$

Если такой элемент существует для любых таких подпространств $Y_{n}$ и чисел $d_{n}$, то говорят, что пространство $X$ обладает (В)-свойством.

Кроме гильбертова пространства, до сих пор неизвестно ни одного примера пространства $X$, обладающего (В)-свойством.

В работе изложена история задачи (1) (см. введение) и новые результаты, решающие ее при дополнительных условиях на числа $d_{n}$ или на подпространства $Y_{n}$ (см. теоремы 1, 2 и следствие 3).

1. Введение. История задачи (1) восходит к С.Н. Бернштейну [1], который положительно решил ее в случае, когда $X$ есть пространство $C[a ; b]$ действительнозначных функций, непрерывных на отрезке $[a ; b]$, с равномерной нормой, а $Y_{n}$ при каждом $n=0,1,2, \ldots$ является подпространством алгебраических многочленов

Работа выполнена при поддержке Российского фонда фундаментальных исследований, грант № 05-01-00962.

(C) П. А. Бородин, 2006 
степени не выше $n$. А.Ф. Тиман ([2; гл. 2, п. 2.5]) без особых усилий перенес доказательство Бернштейна на более общий случай произвольного банахова пространства $X$ и системы строго вложенных конечномерных подпространств $Y_{1} \subset Y_{2} \subset \ldots$. Основной составляющей этого доказательства является следующее утверждение.

Лемма А (Бернштейн, Тиман). Пусть $X$ - произвольное банахово пространство, $Y_{1} \subset Y_{2} \subset \cdots \subset Y_{n} \subset X$ - конечная система строго вложенных подпространств, $d_{1}>d_{2}>\cdots>d_{n} \geqslant 0, u z-$ произвольный элемент из $X \backslash Y_{n}$. Тогда существует элемент $x \in X$, для которого $\rho\left(x, Y_{k}\right)=d_{k}, k=1, \ldots, n,\|x\| \leqslant d_{1}+1$, $u x-\lambda z \in Y_{n}$ для некоторого $\lambda>0$.

В случае, когда все $Y_{k}$ - подпространства существования (в частности, когда все $Y_{k}$ конечномерны или когда $X$ рефлексивно), это утверждение справедливо и для любого набора чисел $d_{1} \geqslant d_{2} \geqslant \cdots \geqslant d_{n} \geqslant 0$.

Напомним, что подпространство $Y \subset X$ называется подпространством существования, если для любого $x \in X$ в $Y$ существует элемент $y$, ближайший к $x$ : $\|x-y\|=\rho(x, Y)$.

ДоКАЗАтЕЛЬСтво. Положим $\varepsilon=\min \left\{d_{n}-d_{n-1}, \ldots, d_{2}-d_{1}, 1\right\}, x_{n}=z$, и выберем произвольные элементы $x_{k} \in Y_{k+1} \backslash Y_{k}, k=1, \ldots, n-1$. Для $\lambda_{n}=d_{n} / \rho\left(x_{n}, Y_{n}\right)$ имеем $\rho\left(\lambda_{n} x_{n}, Y_{n}\right)=d_{n}$. В подпространстве $Y_{n}$ найдется такой элемент $y_{n}$, что $\left\|\lambda_{n} x_{n}-y_{n}\right\| \leqslant d_{n}+\varepsilon$. Функция $f(\lambda):=\rho\left(\lambda_{n} x_{n}-y_{n}+\lambda x_{n-1}, Y_{n-1}\right)$ непрерывна при $\lambda \in$ $[0 ; \infty), f(0) \leqslant\left\|\lambda_{n} x_{n}-y_{n}\right\| \leqslant d_{n}+\varepsilon \leqslant d_{n-1}$, и $f(\lambda) \rightarrow \infty$ при $\lambda \rightarrow \infty$, поэтому найдется такое $\lambda_{n-1} \in[0 ; \infty]$, что $f\left(\lambda_{n-1}\right)=d_{n-1}$, т.е. $\rho\left(\lambda_{n} x_{n}-y_{n}+\lambda_{n-1} x_{n-1}, Y_{n-1}\right)=d_{n-1}$. В подпространстве $Y_{n-1}$ найдется такой элемент $y_{n-1}$, что $\| \lambda_{n} x_{n}-y_{n}+\lambda_{n-1} x_{n-1}-$ $y_{n-1} \| \leqslant d_{n-1}+\varepsilon$. При этом $\rho\left(\lambda_{n} x_{n}-y_{n}+\lambda_{n-1} x_{n-1}-y_{n-1}, Y_{n}\right)=\rho\left(\lambda_{n} x_{n}, Y_{n}\right)=d_{n}$ и $\rho\left(\lambda_{n} x_{n}-y_{n}+\lambda_{n-1} x_{n-1}-y_{n-1}, Y_{n-1}\right)=\rho\left(\lambda_{n} x_{n}-y_{n}+\lambda_{n-1} x_{n-1}, Y_{n-1}\right)=d_{n-1}$. Повторяя эту процедуру еще $n-2$ раза, получим элемент $x=\lambda_{n} x_{n}-y_{n}+\lambda_{n-1} x_{n-1}-$ $y_{n-1}+\cdots+\lambda_{2} x_{2}-y_{2}+\lambda_{1} x_{1}-y_{1}$, для которого $\rho\left(x, Y_{k}\right)=d_{k}, k=1, \ldots, n,\|x\| \leqslant d_{1}+\varepsilon \leqslant$ $d_{1}+1$, и $x-\lambda_{n} z \in Y_{n}$. При этом, если все $Y_{k}$ - подпространства существования и $\varepsilon=0$, то на каждом шаге в качестве $y_{k} \in Y_{k}$ берется элемент, ближайший в $Y_{k} \mathrm{k}$ элементу $\lambda_{n} x_{n}-y_{n}+\cdots-y_{k+1}+\lambda_{k} x_{k}$.

Лемма А доказана.

Теорема Бернштейна-Тимана легко следует из леммы А: если $Y_{1} \subset Y_{2} \subset \cdots-$ счетная система строго вложенных конечномерных подпространств в $X$ и $d_{1} \geqslant$ $d_{2} \geqslant \cdots, d_{n} \rightarrow 0$, то по лемме А строится последовательность элементов $x_{m} \in Y_{m+1}$ с $\rho\left(x_{m}, Y_{k}\right)=d_{k}, k=1, \ldots, m$, и $\left\|x_{m}\right\| \leqslant d_{1}+1, m=1,2, \ldots$. Пусть $y_{k m}$ - элемент $Y_{k}$, ближайший к $x_{m}$. При каждом фиксированном $k$ последовательность $\left\{y_{k m}\right\}_{m=k}^{\infty}$ предкомпактна в силу конечномерности $Y_{k}$ и того, что $\left\|y_{k m}\right\| \leqslant\left\|x_{m}\right\|+\left\|x_{m}-y_{k m}\right\| \leqslant$ $d_{1}+1+d_{k} \leqslant 2 d_{1}+1$. Применяя диагональный процесс, найдем такую последовательность $\Lambda \subset \mathbb{N}$, что при каждом $k$ последовательность $\left\{y_{k m}\right\}_{m \in \Lambda}$ фундаментальна. Соответствующая последовательность $\left\{x_{m}\right\}_{m \in \Lambda}$ также фундаментальна в силу оценки

$$
\left\|x_{m}-x_{l}\right\| \leqslant\left\|x_{m}-y_{k m}\right\|+\left\|x_{l}-y_{k l}\right\|+\left\|y_{k m}-y_{k l}\right\|=2 d_{k}+\left\|y_{k m}-y_{k l}\right\|,
$$

и для предельного элемента $x$ имеем $\rho\left(x, Y_{k}\right)=d_{k}, k=1,2, \ldots$.

Как видно из этого рассуждения и доказательства леммы А, элементы $x_{m}$ с $\rho\left(x_{m}, Y_{k}\right)=d_{k}, k=1, \ldots, m$, могут строиться по-разному и без предположения 
конечномерности подпространств $Y_{k}$, и основная проблема при решении общей задачи (1) методом Бернштейна-Тимана состоит в доказательстве предкомпактности последовательности $\left\{x_{m}\right\}$.

В 1963 г. В.Н. Никольский [3] заметил, что для справедливости основного утверждения леммы А с произвольными $d_{1} \geqslant d_{2} \geqslant \cdots \geqslant d_{n}$ пространство $X$ необходимо должно быть рефлексивным. Более точно, если для любой пары подпространств $Y_{0} \subset Y_{1} \subset X, Y_{0}=\{0\}, Y_{1}=\operatorname{ker} f:=\{y \in X: f(y)=0\}$, где $f \in X^{*}$ - некоторый ненулевой линейный непрерывный функционал на $X$, существует элемент $x \in X$ с $\rho\left(x, Y_{0}\right)=\rho\left(x, Y_{1}\right)=1$, то $X$ рефлексивно. Действительно, $\rho\left(x, Y_{0}\right)=\|x\|$, $\rho\left(x, Y_{1}\right)=f(x) /\|f\|$, и равенство $1=\|x\|=f(x) /\|f\|$ означает, что функционал $f$ достигает своей нормы на элементе $x$. Если же каждый функционал $f \in X^{*}$ достигает своей нормы, то $X$ рефлексивно по теореме Джеймса [4]. Это замечание В. Н. Никольского показывает, что в случае произвольного $X$ и произвольных $Y_{n}$ можно надеяться на положительное решение задачи (1) только в случае строго монотонной последовательности уклонений $d_{1}>d_{2}>\cdots$. Именно, если в нерефлексивном пространстве $X$ взять нерефлексивное подпространство $Y_{2}$ с бесконечной коразмерностью, в $Y_{2}$ взять подпространство $Y_{1}$ - ядро некоторого функционала из $Y_{2}^{*}$, не достигающего своей нормы на $Y_{2}$, в качестве $Y_{0}$ взять $\{0\}$, а в качестве $Y_{3} \subset Y_{4} \subset \cdots-$ произвольные подпространства, содержащие $Y_{2}$, то для системы подпространств $\left\{Y_{n}\right\}_{n=0}^{\infty}$ и чисел $d_{0}=d_{1}=1, d_{2}=d_{3}=\cdots=0$ задача (1) не имеет решения.

В то же время И. С. Тюремских [5] доказал, что в гилъбертовом пространстве $X$ задача (1) имеет положительное решение для любых $Y_{n}$ и $d_{n}$ (см. также следствие 2 ниже).

Шапиро [6] показал, что в любом бесконечномерном пространстве $X$ для любых подпространств $Y_{1} \subset Y_{2} \subset \cdots$ и любых чисел $d_{1} \geqslant d_{2} \geqslant \ldots, d_{n} \rightarrow 0$, существует такой элемент $x$, что $\rho\left(x, Y_{n}\right) \neq O\left(d_{n}\right), n \rightarrow \infty$.

Этот результат был усилен Тюремских [7], который при тех же предположениях установил существование такого элемента $x \in X$, что $\rho\left(x, Y_{n}\right) \geqslant d_{n}, n=1,2, \ldots$. Приведем свое доказательство этого утверждения. Если в $X$ существует такое подпространство $Z$, что $Y_{1} \subset Z$ и $0<\operatorname{dim}\left(Y_{k} / Z\right)<\infty$ при каждом $k \geqslant 2$, то согласно теореме Бернштейна-Тимана в факторпространстве $X / Z$ найдется такой класс смежности $[x]$, что $\rho\left([x], Y_{n} / Z\right)=d_{n}, n=1,2, \ldots$. Тогда для элемента $x$ имеем $\rho\left(x, Y_{n}\right)=\inf \left\{\|x-y\|: y \in Y_{n}\right\} \geqslant \inf \left\{\|x-y-z\|: y \in Y_{n}, z \in Z\right\}=\rho\left([x], Y_{n} / Z\right)=d_{n}$, т.е. $\rho\left(x, Y_{n}\right) \geqslant d_{n}, n=1,2, \ldots$. Что касается подпространства $Z$, то его можно построить следующим образом. Возьмем какие-нибудь элементы $y_{n} \in Y_{n} \backslash Y_{n-1}$, $n=2,3, \ldots$, и построим с помощью теоремы Хана-Банаха такие функционалы $f_{n} \in X^{*}$, что $f_{n}\left(y_{n}\right)>0$ и $Y_{n-1} \subset \operatorname{ker} f_{n}, n=2,3, \ldots$ Положим $Z:=\bigcap_{n=2}^{\infty} \operatorname{ker} f_{n}$. Имеем $Y_{1} \subset Z$, а для $k \geqslant 2$ имеем $Y_{k} \subset \operatorname{ker} f_{n}$ при $n \geqslant k+1$, поэтому $\operatorname{dim} Y_{k} / Z=$ $\operatorname{dim}\left(Y_{k} / \bigcap_{n=2}^{k} \operatorname{ker} f_{n}\right)<\infty$. В то же время $y_{k} \notin Z$ и $y_{k} \in Y_{k}$, так что $\operatorname{dim}\left(Y_{k} / Z\right) \geqslant 1$. Доказательство закончено.

Этот результат Тюремских показывает, что пример отрицательного решения задачи (1) в случае строго монотонной последовательности $d_{n}$ невозможно построить, "играя" на медленном убывании этой последовательности. Как показывают теорема 1 и следствие 1 (см. ниже), для построения такого примера не подойдут и быстро убывающие последовательности. 
Отметим, что теорема Бернштейна-Тимана (случай конечномерных подпространств $Y_{n}$ ) обобщалась на различные классы линейных метрических пространств (см. работы [8], [9] и библиографию в них). Кроме того, в последнее время задача (1) решается в случае нелинейных множеств $Y_{n}$ - например, множеств рациональных функций степени не выше $n$ [10], [11].

\section{2. Случай быстро убывающих уклонений $d_{n}$.}

ТЕОРема 1. Пусть $X$ - произвольное бесконечномерное банахово пространство, $Y_{1} \subset Y_{2} \subset \cdots-$ произвольная счетная система строго вложенных подпространств в $X$, а числовая последовательность $\left\{d_{n}\right\}_{n=1}^{\infty}$ такова, что $d_{n}>\sum_{k=n+1}^{\infty} d_{k}$ для каждого натурального $n \geqslant n_{0}$, при котором $d_{n}>0$. Тогда существует элемент $x \in X$, для которого $\rho\left(x, Y_{n}\right)=d_{n}, n=1,2, \ldots$.

ДокАЗАТЕЛЬСтво. Случай произвольного $n_{0}$ легко сводится к случаю $n_{0}=1$ : умея строить элемент $z$ с $\rho\left(z, Y_{n}\right)=d_{n}$ при $n \geqslant n_{0}$, мы по лемме А построим элемент $x$ с $\rho\left(x, Y_{k}\right)=d_{k}$ при $k=1, \ldots, n_{0}$ и такой, что $x-\lambda z \in Y_{n_{0}}$ при некотором $\lambda>0$. Но тогда $d_{n_{0}}=\rho\left(x, Y_{n_{0}}\right)=\rho\left(\lambda z, Y_{n_{0}}\right)=\lambda d_{n_{0}} \Longrightarrow \lambda=1$ и $\rho\left(x, Y_{n}\right)=d_{n}$ при всех $n \geqslant n_{0}$.

Ниже считаем $n_{0}=1$.

Если $d_{n}=0$ для какого-то $n$, то искомый элемент существует по лемме А, примененной к пространству $Y_{n}$ и подпространствам $Y_{1} \subset Y_{2} \subset \cdots \subset Y_{n-1}$ в нем. Поэтому ниже считаем $d_{n}>0$ при всех $n$.

Для каждого натурального $n$ положим

$$
\varepsilon_{n}=\min _{1 \leqslant k \leqslant n}\left\{d_{k}-\sum_{j=k+1}^{\infty} d_{j}\right\} .
$$

В силу нашего предположения $\varepsilon_{n}>0$, и по лемме А найдутся такие элементы $q_{n} \in Y_{n+1} \backslash Y_{n}$, что $\rho\left(q_{n}, Y_{n}\right)=1$ и

$$
\left\|q_{n}\right\|=\rho\left(q_{n},\{0\}\right)=\frac{d_{n}+\varepsilon_{n} / 2^{n}}{d_{n}}<2 .
$$

Кроме того, найдется такой функционал $f_{n} \in Y_{n}^{\perp}=\left\{f \in X^{*}: f(y)=0 \forall y \in Y_{n}\right\}$, что $\left\|f_{n}\right\|=1=f\left(q_{n}\right)$ (см. [12; гл. 2, п. 3, лемма 12]).

Положим $\lambda_{n}^{n}=d_{n}$. Очевидно, $\rho\left(\lambda_{n}^{n} q_{n}, Y_{n}\right)=d_{n}$. Пусть, для определенности, $f_{n-1}\left(q_{n}\right) \geqslant 0$. Тогда

$$
\rho\left(\lambda_{n}^{n} q_{n}, Y_{n-1}\right) \leqslant\left\|\lambda_{n}^{n} q_{n}\right\|=d_{n}+\frac{\varepsilon_{n}}{2^{n}}<d_{n-1},
$$

а с другой стороны,

$$
\rho\left(\lambda_{n}^{n} q_{n}+d_{n-1} q_{n-1}, Y_{n-1}\right) \geqslant f_{n-1}\left(\lambda_{n}^{n} q_{n}+d_{n-1} q_{n-1}\right) \geqslant d_{n-1},
$$

и потому найдется такое число $\lambda_{n-1}^{n} \in\left[0 ; d_{n-1}\right]$, что $\rho\left(\lambda_{n}^{n} q_{n}+\lambda_{n-1}^{n} q_{n-1}, Y_{n-1}\right)=d_{n-1}$. (Если $f_{n-1}\left(q_{n}\right)<0$, то нужное число $\lambda_{n-1}^{n}$ найдется на отрезке $\left.\left[-d_{n-1} ; 0\right].\right)$ В то же время $\rho\left(\lambda_{n}^{n} q_{n}+\lambda_{n-1}^{n} q_{n-1}, Y_{n}\right)=\rho\left(\lambda_{n}^{n} q_{n}, Y_{n}\right)=d_{n}$. 
Пусть уже найдены такие числа $\lambda_{n}^{n} \in\left[-d_{n} ; d_{n}\right], \ldots, \lambda_{k}^{n} \in\left[-d_{k} ; d_{k}\right]$, что $\rho\left(z_{k}:=\right.$ $\left.\lambda_{n}^{n} q_{n}+\cdots+\lambda_{k}^{n} q_{k}, Y_{m}\right)=d_{m}$ при всех $m=k, \ldots, n$. Пусть для определенности $f_{k-1}\left(z_{k}\right) \geqslant 0$. Тогда

$$
\begin{aligned}
\rho\left(z_{k}, Y_{k-1}\right) & \leqslant\left\|z_{k}\right\| \leqslant \sum_{j=k}^{n}\left|\lambda_{j}^{n}\right|\left\|q_{j}\right\| \leqslant \sum_{j=k}^{n} d_{j} \frac{d_{j}+\varepsilon_{j} / 2^{j}}{d_{j}} \\
& \leqslant \sum_{j=k}^{n} d_{j}+\varepsilon_{k-1} \sum_{j=k}^{n} \frac{1}{2^{j}}<\sum_{j=k}^{n} d_{j}+\varepsilon_{k-1} \leqslant d_{k-1},
\end{aligned}
$$

а с другой стороны,

$$
\rho\left(z_{k}+d_{k-1} q_{k-1}, Y_{k-1}\right) \geqslant f_{k-1}\left(z_{k}+d_{k-1} q_{k-1}\right) \geqslant d_{k-1},
$$

и потому найдется такое число $\lambda_{k-1}^{n} \in\left[0 ; d_{k-1}\right]$, что $\rho\left(z_{k}+\lambda_{k-1}^{n} q_{k-1}, Y_{k-1}\right)=d_{k-1}$. (Если $f_{k-1}\left(z_{k}\right)<0$, то нужное число $\lambda_{k-1}^{n}$ найдется на отрезке $\left.\left[-d_{k-1} ; 0\right].\right)$ В то же время $\rho\left(z_{k}+\lambda_{k-1}^{n} q_{k-1}, Y_{m}\right)=\rho\left(z_{k}, Y_{m}\right)=d_{m}$ при каждом $m=k, \ldots, n$.

Продолжая этот процесс до $k=1$ включительно, получим элемент $x_{n}=\lambda_{n}^{n} q_{n}+$ $\cdots+\lambda_{1}^{n} q_{1}$, для которого $\rho\left(x_{n}, Y_{k}\right)=d_{k}$ и $\left|\lambda_{k}^{n}\right| \leqslant d_{k}$ при каждом $k=1, \ldots, n$. С помощью обычного диагонального процесса выберем последовательность $\Lambda$ номеров $n$, для которых при каждом натуральном $k$ имеет место сходимость $\lambda_{k}^{n} \rightarrow \lambda_{k}$ при $n \rightarrow \infty, n \in \Lambda$. Имеем $\left|\lambda_{k}\right| \leqslant d_{k}$, и ряд $\sum_{k=1}^{\infty} \lambda_{k} q_{k}$ сходится по норме пространства $X$ :

$$
\left\|\sum_{k=m}^{n} \lambda_{k} q_{k}\right\| \leqslant \sum_{k=m}^{n}\left|\lambda_{k}\right|\left\|q_{k}\right\| \leqslant \sum_{k=m}^{n} d_{k} \cdot 2 \leqslant 2 d_{m-1} \rightarrow 0, \quad m, n \rightarrow \infty .
$$

Элемент $x=\sum_{k=1}^{\infty} \lambda_{k} q_{k}$ является пределом последовательности $\left\{x_{n}\right\}_{n \in \Lambda}$ :

$$
\begin{aligned}
\left\|x-x_{n}\right\| & \leqslant \sum_{k=1}^{n}\left|\lambda_{k}^{n}-\lambda_{k}\right|\left\|q_{k}\right\|+\sum_{k=n+1}^{\infty}\left|\lambda_{k}\right|\left\|q_{k}\right\| \\
& \leqslant \sum_{k=1}^{N}\left|\lambda_{k}^{n}-\lambda_{k}\right|\left\|q_{k}\left|+\sum_{k=N+1}^{n}\left(\left|\lambda_{k}^{n}\right|+\left|\lambda_{k}\right|\right)\left\|q_{k}\right\|+\sum_{k=n+1}^{\infty}\right| \lambda_{k} \mid\right\| q_{k} \| \\
& \leqslant 2 \sum_{k=1}^{N}\left|\lambda_{k}^{n}-\lambda_{k}\right|+\sum_{k=N+1}^{n} 2 d_{k} \cdot 2+\sum_{k=n+1}^{\infty} d_{k} \cdot 2 \\
& \leqslant 2 N \max _{1 \leqslant k \leqslant N}\left|\lambda_{k}^{n}-\lambda_{k}\right|+4 d_{N} \rightarrow 0, \quad n \rightarrow \infty, \quad n \in \Lambda, \quad N=N(n) \rightarrow \infty .
\end{aligned}
$$

Следовательно, $\rho\left(x, Y_{k}\right)=\lim _{\Lambda \ni n \rightarrow \infty} \rho\left(x_{n}, Y_{k}\right)=d_{k}, k=1,2, \ldots$.

Теорема 1 доказана.

СЛЕДСТВИЕ 1. Для любой системы подпространств $Y_{1} \subset Y_{2} \subset \cdots$ бесконечномерного банахова пространства $X$ и любой последовательности $d_{1} \geqslant d_{2} \geqslant \cdots$, $d_{n} \rightarrow 0$, существует такой элемент $x \in X$, что $\rho\left(x, Y_{n}\right) \leqslant d_{n}$, причем $d_{n}>0 \Longrightarrow$ $\rho\left(x, Y_{n}\right)>0, n=1,2, \ldots$. 
ДокАЗАТЕЛЬСтво. Последовательность $h_{1}:=d_{1}, h_{n}:=\min \left\{h_{n-1} / 3, d_{n}\right\}, n=$ $2,3, \ldots$, при каждом $n$ удовлетворяет условиям: $h_{n} \leqslant d_{n}, d_{n}>0 \Longrightarrow h_{n}>0$ и

$$
\sum_{k=n+1}^{\infty} h_{k} \leqslant \sum_{k=n+1}^{\infty} \frac{h_{n}}{3^{k-n}} \leqslant h_{n}
$$

причем знак последнего неравенства строгий в случае $h_{n}>0$.

Применяя теорему 1 к системе $\left\{Y_{k}\right\}_{k=1}^{\infty}$ и последовательности $\left\{h_{k}\right\}_{k=1}^{\infty}$, получаем требуемый элемент $x$.

\section{3. Дополнительные условия на подпространства $Y_{n}$.}

Лемма 1. Пусть $d_{1} \geqslant d_{2} \geqslant \cdots \geqslant d_{n}>0$, а система строго вложенных подпространств $Y_{1} \subset Y_{2} \subset \cdots \subset Y_{n}$ банахова пространства $X$ удовлетворяет условию: существуют такие ненулевые элементы $q_{n} \in X \backslash Y_{n}, q_{k} \in Y_{k+1} \backslash Y_{k}, k=$ $1, \ldots, n-1$, что для любого ненулевого элемента $q \in\left\langle q_{k}, \ldots, q_{n}\right\rangle$ (из линейной оболочки $\left.q_{k}, \ldots, q_{n}\right)$ выполняется неравенство

$$
\frac{\rho\left(q, Y_{k}\right)}{\rho\left(q, Y_{k-1}\right)} \geqslant \frac{d_{k}}{d_{k-1}}, \quad k=2, \ldots, n .
$$

Тогда в подпространстве $\left\langle q_{1}, \ldots, q_{n}\right\rangle$ найдется такой элемент $x$, что $\rho\left(x, Y_{k}\right)=d_{k}$, $k=1, \ldots, n$.

ДокАЗАТЕЛЬСТВо проведем индукцией по $n$.

При $n=1$ в качестве искомого можно взять элемент

$$
x=\frac{d_{1}}{\rho\left(q_{1}, Y_{1}\right)} q_{1} .
$$

При $n=2$ для любого элемента

$$
x(\lambda)=\frac{d_{2}}{\rho\left(q_{2}, Y_{2}\right)} q_{2}+\lambda q_{1}
$$

имеем

$$
\rho\left(x(\lambda), Y_{2}\right)=\rho\left(\frac{d_{2}}{\rho\left(q_{2}, Y_{2}\right)} q_{2}, Y_{2}\right)=d_{2}
$$

С другой стороны,

$$
\rho\left(x(0), Y_{1}\right)=\rho\left(\frac{d_{2}}{\rho\left(q_{2}, Y_{2}\right)} q_{2}, Y_{1}\right)=\frac{d_{2}}{\rho\left(q_{2}, Y_{2}\right)} \rho\left(q_{2}, Y_{1}\right) \leqslant d_{1}
$$

и $\rho\left(x(\lambda), Y_{1}\right) \rightarrow \infty$ при $\lambda \rightarrow \infty$, поэтому найдется такое число $\lambda_{0}$, что $\rho\left(x\left(\lambda_{0}\right), Y_{1}\right)=$ $d_{1}$.

В случае $n \geqslant 3$ для любого ненулевого элемента $u \in\left\langle q_{n-1}, q_{n}\right\rangle$ и любого элемента $q \in\left\langle q_{k}, \ldots, q_{n-2}, u\right\rangle$ имеем

$$
\frac{\rho\left(q, Y_{k}\right)}{\rho\left(q, Y_{k-1}\right)} \geqslant \frac{d_{k}}{d_{k-1}}, \quad k=2, \ldots, n-2, \quad \frac{\rho\left(u, Y_{n-1}\right)}{\rho\left(u, Y_{n-2}\right)} \geqslant \frac{d_{n-1}}{d_{n-2}},
$$

поэтому система $q_{1}, \ldots, q_{n-2}, u$ удовлетворяет условиям леммы в отношении подпространств $Y_{1}, \ldots, Y_{n-1}$. По предположению индукции существует такой элемент 
$x(u) \in\left\langle q_{1}, \ldots, q_{n-2}, u\right\rangle$, что $\rho\left(x(u), Y_{k}\right)=d_{k}, k=1, \ldots, n-1$. Обозначим через $\lambda(u)$ число, для которого $x(u)-\lambda(u) u \in\left\langle q_{1}, \ldots, q_{n-2}\right\rangle$. Очевидно, $\lambda(u)=d_{n-1} / \rho\left(u, Y_{n-1}\right)$, т.е. $\lambda(u)$ непрерывно зависит от $u$. Следовательно, функция $\rho\left(x(u), Y_{n}\right)=\rho\left(\lambda(u) u, Y_{n}\right)$ тоже непрерывно зависит от $u$. При этом $\rho\left(x\left(q_{n-1}\right), Y_{n}\right)=0$, a

$$
\rho\left(x\left(q_{n}\right), Y_{n}\right)=\rho\left(\lambda\left(q_{n}\right) q_{n}, Y_{n}\right)=\frac{d_{n-1}}{\rho\left(q_{n}, Y_{n-1}\right)} \rho\left(q_{n}, Y_{n}\right) \geqslant d_{n},
$$

поэтому найдется такой элемент $u_{n} \in\left\langle q_{n-1}, q_{n}\right\rangle$, что для соответствующего элемента $x=x\left(u_{n}\right)$ выполняется дополнительное равенство $\rho\left(x, Y_{n}\right)=d_{n}$.

Лемма 1 доказана.

Теорема 2. Пусть $d_{0} \geqslant d_{1} \geqslant d_{2} \geqslant \ldots, d_{n} \rightarrow 0$, а счетная система строго вложенных подпространств $Y_{1} \subset Y_{2} \subset \ldots$ бесконечномерного банахова пространства $X$ удовлетворяет условию: существуют такие ненулевые элементы $q_{n} \in$ $Y_{n+1} \backslash Y_{n}, n=1,2, \ldots$, что для любого $k \geqslant 1$ и любого элемента $q \in\left\langle q_{k}, q_{k+1}, \ldots\right\rangle$ (из линейной оболочки $q_{k}, q_{k+1}, \ldots$ ) выполняется неравенство

$$
\|q\| \leqslant \frac{d_{k-1}}{d_{k}} \rho\left(q, Y_{k}\right) .
$$

Тогда в замыкании $\overline{\left\langle q_{1}, q_{2}, \ldots\right\rangle}$ линейной оболочки элементов $q_{n}$ найдется такой элемент $x$, что $\rho\left(x, Y_{n}\right)=d_{n}, n=1,2, \ldots$.

ДокАзАтЕльство. Для любых натуральных чисел $2 \leqslant k<n$ и любого ненулевого элемента $q \in\left\langle q_{k}, \ldots, q_{n}\right\rangle$ получаем

$$
\frac{\rho\left(q, Y_{k}\right)}{\rho\left(q, Y_{k-1}\right)} \geqslant \frac{\rho\left(q, Y_{k}\right)}{\|q\|} \geqslant \frac{d_{k}}{d_{k-1}} .
$$

Следовательно, по лемме 1 найдутся такие элементы $x_{n} \in\left\langle q_{1}, \ldots, q_{n}\right\rangle$, что $\rho\left(x, Y_{k}\right)=$ $d_{k}, k=1, \ldots, n$.

Покажем, что из последовательности $x_{n}$ можно извлечь сходящуюся подпоследовательность. Пусть $x_{n}=y_{k}^{n}+v_{k}^{n}$, где $k<n, y_{k}^{n} \in\left\langle q_{1}, \ldots, q_{k-1}\right\rangle, v_{k}^{n} \in\left\langle q_{k}, q_{k+1}, \ldots\right\rangle$. Имеем

$$
\begin{aligned}
& \left\|v_{k}^{n}\right\| \leqslant \frac{d_{k-1}}{d_{k}} \rho\left(v_{k}^{n}, Y_{k}\right)=\frac{d_{k-1}}{d_{k}} \rho\left(x_{n}, Y_{k}\right)=d_{k-1}, \\
& \left\|y_{k}^{n}\right\| \leqslant\left\|x_{n}\right\|+\left\|v_{k}^{n}\right\| \leqslant \frac{d_{0}}{d_{1}} \rho\left(x_{n}, Y_{1}\right)+d_{k-1}=d_{0}+d_{k-1} .
\end{aligned}
$$

$\mathrm{C}$ помощью диагонального процесса выберем такую последовательность $\Lambda \subset \mathbb{N}$, чтобы для каждого фиксированного $k$ имела место сходимость $y_{k}^{n} \rightarrow y_{k}, n \rightarrow \infty$, $n \in \Lambda$ (при каждом фиксированном $k$ элементы $\left\{y_{k}^{n}\right\}_{n=1}^{\infty}$ ограничены и лежат в конечномерном подпространстве). Тогда при $n, m \in \Lambda$ и $k<\min \{n, m\}$ получаем

$$
\begin{aligned}
\left\|x_{n}-x_{m}\right\| & \leqslant\left\|y_{k}^{n}-y_{k}^{m}\right\|+\left\|v_{k}^{n}\right\|+\left\|v_{k}^{m}\right\| \\
& \leqslant\left\|y_{k}^{n}-y_{k}^{m}\right\|+2 d_{k-1} \rightarrow 0, \quad n, m, k(n, m) \rightarrow \infty,
\end{aligned}
$$

т.е. последовательность $\left\{x_{n}\right\}_{n \in \Lambda}$ фундаментальна и сходится к искомому элементу $x$.

Теорема 2 доказана. 
СлЕДСтвиЕ 2 (Тюремских [5]). В гилъбертовом пространстве $X$ задача (1) решается положительно для любых $Y_{n} u d_{n}$.

ДоКАЗАТЕЛЬСтво. Возьмем в качестве $q_{k}$ любой ненулевой элемент из подпространства $Y_{k+1}$, ортогональный подпространству $Y_{k}$. Тогда для любого элемента $q \in\left\langle q_{k}, q_{k+1}, \ldots\right\rangle$ имеем $q \perp Y_{k}$ и

$$
\|q\|=\rho\left(q, Y_{k}\right) \leqslant \frac{d_{k-1}}{d_{k}} \rho\left(q, Y_{k}\right),
$$

так что выполнены все условия теоремы 2 (число $d_{0}$ можно положить равным $d_{1}$ ).

СЛЕДСТВиЕ 3. Пусть $X$ есть одно из пространств $c_{0}$ или $\ell_{p}, 1<p<\infty$, $d_{1}>d_{2}>\cdots, d_{n} \rightarrow 0$, а система строго вложенных подпространств $Y_{1} \subset Y_{2} \subset \cdots$ такова, что для любого $n \geqslant n_{0}$ размерность факторпространства $Y_{n+1} / Y_{n}$ бесконечна. Тогда в $X$ существует такой элемент $x$, что $\rho\left(x, Y_{n}\right)=d_{n}, n=1,2, \ldots$.

ДоКАЗАТЕЛЬСТво. Случай произвольного $n_{0}$ легко сводится к случаю $n_{0}=1$ : умея строить элемент $z$ с $\rho\left(z, Y_{n}\right)=d_{n}$ при $n \geqslant n_{0}$, мы по лемме А построим элемент $x$ с $\rho\left(x, Y_{k}\right)=d_{k}$ при $k=1, \ldots, n_{0}$ и такой, что $x-\lambda z \in Y_{n_{0}}$ при некотором $\lambda>0$. Но тогда $d_{n_{0}}=\rho\left(x, Y_{n_{0}}\right)=\rho\left(\lambda z, Y_{n_{0}}\right)=\lambda d_{n_{0}} \Longrightarrow \lambda=1$ и $\rho\left(x, Y_{n}\right)=\rho\left(z, Y_{n}\right)=d_{n}$ при всех $n \geqslant n_{0}$.

Дальнейшие рассуждения проведем в случае $n_{0}=1$ и $X=c_{0}$.

Построим индуктивно элементы $q_{n}$, требуемые в теореме 2 .

Положим $d_{0}=2 d_{1}$,

$$
\varepsilon_{k}=\min _{1 \leqslant j \leqslant k} \frac{d_{j-1}-d_{j}}{4 d_{j-1}}, \quad k=1,2, \ldots .
$$

Возьмем произвольный элемент $q_{1} \in Y_{2} \backslash Y_{1}$ со свойствами $\left\|q_{1}\right\|=1, \rho\left(q_{1}, Y_{1}\right)>1-$ $\varepsilon_{1}$. Существует такой функционал $f_{1} \in Y_{1}^{\perp} \subset c_{0}^{*}=\ell_{1}$, что $\left\|f_{1}\right\|=1$ и $f_{1}\left(q_{1}\right)>1-\varepsilon_{1}$. Выберем такой номер $n_{1}$, что для координатного проектора $\pi_{1}:\left(x^{1}, x^{2}, \ldots, x^{n_{1}}, \ldots\right)$ $\rightarrow\left(0, \ldots, 0, x^{n_{1}}, x^{n_{1}+1}, \ldots\right)$, действующего в пространствах $c_{0}$ и $\ell_{1}$, имеют место неравенства $\left\|\pi_{1}\left(q_{1}\right)\right\| \leqslant \varepsilon_{1} / 2,\left\|\pi_{1}\left(f_{1}\right)\right\| \leqslant \varepsilon_{1} / 2$.

Подпространство $\pi_{1}\left(c_{0}\right)=\left\{x \in c_{0}: x^{1}=x^{2}=\cdots=x^{n_{1}-1}=0\right\}$ имеет конечную коразмерность в $c_{0}$, а по условию $\operatorname{dim} Y_{3} / Y_{2}=\infty$, поэтому $\operatorname{dim}\left[\left(Y_{3} \cap \pi_{1}\left(c_{0}\right)\right) / \pi_{1}\left(Y_{2}\right)\right]=$ $\infty$, и в подпространстве $Y_{3} \cap \pi_{1}\left(c_{0}\right)$ найдется такой элемент $q_{2}$, что $\left\|q_{2}\right\|=\left\|\pi_{1}\left(q_{2}\right)\right\|=1$ и $\rho\left(q_{2}, Y_{2}\right) \geqslant \rho\left(q_{2}, \pi_{1}\left(Y_{2}\right)\right)>1-\varepsilon_{2}$. Возьмем такой функционал $f_{2} \in Y_{2}^{\perp} \cap \pi_{1}\left(\ell_{1}\right)$, что $\left\|f_{2}\right\|=1$ и $f_{2}\left(q_{2}\right)>1-\varepsilon_{2}$. Выберем такой номер $n_{2}$, что для координатного проектора $\pi_{2}:\left(x^{1}, x^{2}, \ldots, x^{n_{2}}, \ldots\right) \rightarrow\left(0, \ldots, 0, x^{n_{2}}, x^{n_{2}+1}, \ldots\right)$, действующего в пространствах $c_{0}$ и $\ell_{1}$, имеют место неравенства $\left\|\pi_{2}\left(q_{1}\right)\right\| \leqslant \varepsilon_{1} / 2^{2},\left\|\pi_{2}\left(f_{1}\right)\right\| \leqslant \varepsilon_{1} / 2^{2}$, $\left\|\pi_{2}\left(q_{2}\right)\right\| \leqslant \varepsilon_{2} / 2^{2},\left\|\pi_{2}\left(f_{2}\right)\right\| \leqslant \varepsilon_{2} / 2^{2}$.

Продолжая этот процесс по индукции, получим последовательности $\left\{n_{k}\right\}$ натуральных чисел, соответствующих координатных проекторов $\pi_{k}:\left(x^{1}, x^{2}, \ldots, x^{n_{k}}, \ldots\right)$ $\rightarrow\left(0, \ldots, 0, x^{n_{k}}, x^{n_{k}+1}, \ldots\right)$, действующих в пространствах $c_{0}$ и $\ell_{1}$, элементов $q_{k} \in$ $Y_{k+1} \cap \pi_{k-1}\left(c_{0}\right)$, элементов $f_{k} \in Y_{k}^{\perp} \cap \pi_{k-1}\left(\ell_{1}\right)$ со следующими свойствами: $\left\|q_{k}\right\|=1$, $\left\|f_{k}\right\|=1, f_{k}\left(q_{k}\right)>1-\varepsilon_{k} ;\left\|\pi_{k}\left(q_{j}\right)\right\| \leqslant \varepsilon_{j} / 2^{k}, 1 \leqslant j \leqslant k ;\left\|\pi_{k}\left(f_{j}\right)\right\| \leqslant \varepsilon_{j} / 2^{k}, 1 \leqslant j \leqslant k$.

Покажем, что последовательность $\left\{q_{k}\right\}$ удовлетворяет требованиям теоремы 2 . Для этого возьмем произвольную конечную линейную комбинацию $q=\sum_{j=k}^{N} \lambda_{j} q_{j} \mathrm{c}$ нормой $\|q\|=1$ и докажем, что $\rho\left(q, Y_{k}\right) \geqslant d_{k} / d_{k-1}$. 
Для каждого $m=k, \ldots, N$ имеем

$$
\begin{aligned}
1 & =\|q\| \geqslant\left\|\pi_{m-1}(q)-\pi_{m}(q)\right\| \geqslant\left\|\left(\pi_{m-1}-\pi_{m}\right)\left(\lambda_{m} q_{m}\right)\right\| \\
& -\sum_{j \neq m}\left\|\left(\pi_{m-1}-\pi_{m}\right)\left(\lambda_{j} q_{j}\right)\right\|=\left|\lambda_{m}\right|\left\|q_{m}\right\|-\sum_{j=k}^{m-1}\left\|\left(\pi_{m-1}-\pi_{m}\right)\left(\lambda_{j} q_{j}\right)\right\| \\
\geqslant & \left|\lambda_{m}\right|-\sum_{j=k}^{m-1}\left|\lambda_{j}\right|\left\|\pi_{m-1}\left(q_{j}\right)\right\| \geqslant\left|\lambda_{m}\right|-\sum_{j=k}^{m-1}\left|\lambda_{j}\right| \frac{\varepsilon_{j}}{2^{m-1}} \\
\geqslant & \left|\lambda_{m}\right|-\frac{\varepsilon_{1}}{2^{m-1}} \sum_{j=k}^{m-1}\left|\lambda_{j}\right| \geqslant\left|\lambda_{m}\right|-\frac{1}{2^{m+1}} \sum_{j=k}^{m-1}\left|\lambda_{j}\right|,
\end{aligned}
$$

т.е. $\left|\lambda_{k}\right| \leqslant 1$ и

$$
\left|\lambda_{m}\right| \leqslant 1+\frac{1}{2^{m+1}} \sum_{j=k}^{m-1}\left|\lambda_{j}\right|
$$

при $m>k$, откуда по индукции доказывается, что $\left|\lambda_{m}\right|<2$ при любом $m \geqslant k$.

В то же время найдется такое $s \geqslant k$, что $\left\|\left(\pi_{s-1}-\pi_{s}\right)(q)\right\|=\|q\|=1$, откуда

$$
\begin{aligned}
\left|\lambda_{s}\right|= & \left\|\lambda_{s} q_{s}\right\|=\left\|\left(\pi_{s-1}-\pi_{s}\right)\left(\lambda_{s} q_{s}\right)\right\| \geqslant\left\|\left(\pi_{s-1}-\pi_{s}\right)(q)\right\| \\
& -\left\|\sum_{j \neq s}\left(\pi_{s-1}-\pi_{s}\right)\left(\lambda_{j} q_{j}\right)\right\|=1-\left\|\sum_{j=k}^{s-1}\left(\pi_{s-1}-\pi_{s}\right)\left(\lambda_{j} q_{j}\right)\right\| \\
\geqslant & 1-\sum_{j=k}^{s-1}\left\|\pi_{s-1}\left(\lambda_{j} q_{j}\right)\right\| \geqslant 1-\sum_{j=k}^{s-1}\left|\lambda_{j}\right|\left\|\pi_{s-1}\left(q_{j}\right)\right\| \\
\geqslant & 1-\sum_{j=k}^{s-1} 2 \cdot \frac{\varepsilon_{j}}{2^{s-1}} \geqslant 1-\varepsilon_{k} \frac{s-k}{2^{s-2}} \geqslant 1-\varepsilon_{k} \frac{s-1}{2^{s-2}} \geqslant 1-\varepsilon_{k} .
\end{aligned}
$$

Далее, поскольку $f_{s} \in Y_{s}^{\perp} \subseteq Y_{k}^{\perp}$, то

$$
\begin{aligned}
\rho\left(q, Y_{k}\right) & \geqslant\left|f_{s}(q)\right| \geqslant\left|f_{s}\left(\lambda_{s} q_{s}\right)\right|-\sum_{j=k}^{s-1}\left|f_{j}\left(\lambda_{j} q_{j}\right)\right|-\sum_{j=s+1}^{N}\left|f_{s}\left(\lambda_{j} q_{j}\right)\right| \\
& \geqslant\left|\lambda_{s}\right|\left(1-\varepsilon_{k}\right)-\sum_{j=k}^{s-1}\left|\lambda_{j}\right|\left|f_{s}\left(\pi_{s-1}\left(q_{j}\right)\right)\right|-\sum_{j=s+1}^{N}\left|\lambda_{j}\right|\left|\left(\pi_{j-1}\left(f_{s}\right)\right)\left(q_{j}\right)\right| \\
& \geqslant\left(1-\varepsilon_{k}\right)^{2}-\sum_{j=k}^{s-1} 2 \cdot \frac{\varepsilon_{j}}{2^{s-1}}-\sum_{j=s+1}^{N} 2 \cdot \frac{\varepsilon_{s}}{2^{j-1}} \\
& \geqslant\left(1-\varepsilon_{k}\right)^{2}-\varepsilon_{k} \frac{s-k}{2^{s-2}}-\varepsilon_{s} \frac{1}{2^{s-2}} \geqslant\left(1-\varepsilon_{k}\right)^{2}-\varepsilon_{k}\left(\frac{s-1}{2^{s-2}}+\frac{1}{2^{s-2}}\right) \\
& \geqslant\left(1-\varepsilon_{k}\right)^{2}-2 \varepsilon_{k} \geqslant 1-4 \varepsilon_{k} \geqslant 1-4 \frac{d_{k-1}-d_{k}}{4 d_{k-1}}=\frac{d_{k}}{d_{k-1}},
\end{aligned}
$$

что и требовалось. 
4. Некоторые родственные задачи. (а) Если в условии задачи (1) отказаться от требования $\overline{\bigcup_{n=1}^{\infty} Y_{n}}=X$, то получится такая задача: пусть в бесконечномерном банаховом пространстве $X$ задана счетная система строго вложенных линейных подпространств $Y_{1} \subset Y_{2} \subset \ldots$, а также задана последовательность неотрицательных чисел $d_{1} \geqslant d_{2} \geqslant \ldots$ (уже необязательно стремящаяся к нулю). Существует ли элемент $x \in X$, для которого $\rho\left(x, Y_{n}\right)=d_{n}, n=1,2, \ldots$ ?

Следующий пример показывает, что эта задача может не иметь решений для всех последовательностей $\left\{d_{n}\right\}$, не стремящихся к нулю и не становящихся постоянными, начиная с некоторого номера. Пусть $Z$ - произвольное бесконечномерное банахово пространство, $L_{1} \subset L_{2} \subset \ldots$ - строго вложенные линейные подпространства в $Z, \overline{\bigcup_{n=1}^{\infty} L_{n}}=Z$. Положим $X=Z \times \mathbb{R}$ с нормой $\|(z, t)\|=\max \{\|z\|,|t|\}$. Подпространства $Y_{n}=L_{n} \times\{0\}$ образуют строго расширяющуюся систему в $X$. Для любого элемента $x=(z, t) \in X$ имеем $\rho\left(x, Y_{n}\right)=\max \left\{\rho\left(z, L_{n}\right),|t|\right\}$, т.е. либо $\rho\left(x, Y_{n}\right)=\rho\left(z, L_{n}\right) \rightarrow 0$ (при $\left.t=0\right)$, либо $\rho\left(x, Y_{n}\right)=|t|>0$, начиная с некоторого номера $n$.

(б) В задаче (1) вместо счетной системы подпространств $\left\{Y_{n}\right\}$ можно рассматривать континуальную систему $\left\{Y_{\alpha}\right\}_{\alpha \in[0 ; 1]}$ строго вложенных подпространств: $Y_{\alpha} \subset Y_{\beta}$ при $\alpha<\beta$, и искать элемент $x$ с $\rho\left(x, Y_{\alpha}\right)=\rho(\alpha)$ при любом $\alpha \in[0 ; 1]$, где $\rho(\alpha)-$ (вообще говоря, нестрого) убывающая неотрицательная функция на отрезке $[0 ; 1]$.

Следующий пример показывает, что такая задача может не иметь решений для любой функции $\rho(\alpha)$, не являющейся функцией скачков. Пусть $X=\ell_{2}[0 ; 1]$ - пространство функций $f:[0 ; 1] \rightarrow \mathbb{R}$ с конечной суммой $\|f\|^{2}=\sum_{x \in[0 ; 1]}|f(x)|^{2}$ (каждая такая функция отлична от нуля на не более чем счетном числе точек $x_{k} \in[0 ; 1]$, составляющих носитель $\operatorname{supp} f$ функции $f)$. Возьмем в $\ell_{2}[0 ; 1]$ систему строго вложенных подпространств $Y_{\alpha}=\left\{f \in \ell_{2}[0 ; 1]: \operatorname{supp} f \subset[0 ; \alpha]\right\}, \alpha \in[0 ; 1]$. Для любой функции $f \in \ell_{2}[0 ; 1]$ с $\operatorname{supp} f=\left\{x_{k}\right\}$ имеем

$$
\rho\left(f, Y_{\alpha}\right)^{2}=\sum_{\alpha<x_{k} \leqslant 1}\left|f\left(x_{k}\right)\right|^{2}=\sum_{k}\left|f\left(x_{k}\right)\right|^{2} I_{\left[0 ; x_{k}\right)}(\alpha),
$$

где функция $I_{\left[0 ; x_{k}\right)}(t)$ - индикатор промежутка $\left[0 ; x_{k}\right)$.

(в) Тюремских [7] исследовал задачу о существовании элемента с заданными уклонениями от сжимающейся системы строго вложенных подпространств $Y_{1} \supset$ $Y_{2} \supset \cdots$. Им доказана следующая

ТеоремА Б. Пусть $X$ - бесконечномерное банахово пространство. Чтобъ для любой системы строго вложенных подпространств $Y_{1} \supset Y_{2} \supset \cdots$ в $X$ и любой последовательности $d_{1} \leqslant d_{2} \leqslant \cdots$ неотрицательных чисел с $d_{n} \rightarrow d>0$ существовал элемент $x \in X$ с $\rho\left(x, Y_{n}\right)=d_{n}, n=1,2, \ldots, u\|x\|=d$, необходимо и достаточно, чтобы пространство $X$ было рефлексивным.

Необходимость рефлексивности доказывается здесь так же, как в упоминавшемся выше результате Никольского (см. п. 1). В случае же рефлексивного $X$ искомый элемент $x$ может быть построен как элемент из второго сопряженного пространства $X^{* *}=X$, т.е. как функционал на пространстве $X^{*}$, норма которого на подпространстве $Y_{n}^{\perp} \subset X^{*}\left(Y_{1}^{\perp} \subset Y_{2}^{\perp} \subset \cdots\right)$ равна $d_{n}, n=1,2, \ldots$. Это построение аналогично построению в доказательстве теоремы Хана-Банаха: сначала берется функционал на $Y_{1}^{\perp}$ с нормой $d_{1}$, затем он продолжается на $Y_{2}^{\perp}$ с нормой $d_{2}$, и т.д. 


\section{СПИСОК ЦИТИРОВАННОЙ ЛИТЕРАТУРЫ}

[1] С.Н. Бернштейн, "Об обратной задаче теории наилучшего приближения непрерывных функций", Собрание сочинений, 2, Изд-во АН СССР, 1954, 292-294.

[2] А. Ф. Тиман, Теория приближений функиий действительного переменного, Физматгиз, М., 1960.

[3] В.Н.Никольский, "О некоторых свойствах рефлексивных пространств”, Уч. зап. Калин. гос. пед. ин-та, 29 (1963), 121-125.

[4] R. C. James, "Reflexivity and the supremum of linear functionals", Ann. of Math., 66:1 (1957), 159-169.

[5] И. С. Тюремских, “(В)-свойство гильбертовых пространств”, Уч. зап. Калин. гос. пед. ин-та, 39 (1964), 53-64.

[6] H. S. Shapiro, "Some negative theorems on approximation theory", Michigan Math. J., 11:3 (1964), 211-217.

[7] И. С. Тюремских, "Об одной задаче С.Н. Бернштейна", Уч. зап. Калин. гос. пед. инma, 52 (1967), 123-129.

[8] G. Lewicki, "Bernstein's "lethargy" theorem in metrizable topological linear spaces", Monatsh. Math., 113:3 (1992), 213-226.

[9] А. И. Васильев, "Обратная задача теории наилучшего приближения в $F$-пространствах", Докл. РАН, 365:5 (1999), 583-585.

[10] А. А. Пекарский, "Существование функции с заданными наилучшими равномерными рациональными приближениями", Изв. АН Беларуси. Сер. физ.-матем. наук, 1994, № 1, 23-26.

[11] А. П. Старовойтов, "K проблеме описания последовательностей наилучших тригонометрических рациональных приближений", Матем. сб., 191:6 (2000), 145-154.

[12] Н. Данфорд, Дж. Т. Шварц, Линейные операторы. Общая теория, УРСС, М., 2004.

П. А. Бородин

Московский государственный университет

им. М. В. Ломоносова

E-mail: jborodina@inbox.ru
Поступило

24.02.2006 